\title{
Three-dimensional simulations of near-surface convection in main-sequence stars
}

\section{The structure of small-scale magnetic flux concentrations ${ }^{\star}$}

\author{
B. Beeck ${ }^{1}$, M. Schüssler ${ }^{1}$, R. H. Cameron ${ }^{1}$, and A. Reiners ${ }^{2}$ \\ ${ }^{1}$ Max-Planck-Institut für Sonnensystemforschung, Justus-von-Liebig-Weg 3, 37077 Göttingen, Germany \\ e-mail: beeck@mps.mpg.de \\ 2 Institut für Astrophysik, Universität Göttingen, Friedrich-Hund-Platz 1, 37077 Göttingen, Germany
}

Received 2 February 2015 / Accepted 23 April 2015

\section{ABSTRACT}

\begin{abstract}
Context. The convective envelopes of cool main-sequence stars harbour magnetic fields with a complex global and local structure. These fields affect the near-surface convection and the outer stellar atmospheres in many ways and are responsible for the observable magnetic activity of stars.

Aims. Our aim is to understand the local structure in unipolar regions with moderate average magnetic flux density. These correspond to plage regions covering a substantial fraction of the surface of the Sun (and likely also the surface of other Sun-like stars) during periods of high magnetic activity.

Methods. We analyse the results of 18 local-box magnetohydrodynamics simulations covering the upper layers of the convection zones and the photospheres of cool main-sequence stars of spectral types F to early M. The average vertical field in these simulations ranges from 20 to $500 \mathrm{G}$.

Results. We find a substantial variation of the properties of the surface magnetoconvection between main-sequence stars of different spectral types. As a consequence of a reduced efficiency of the convective collapse of flux tubes, M dwarfs lack bright magnetic structures in unipolar regions of moderate field strength. The spatial correlation between velocity and the magnetic field as well as the lifetime of magnetic structures and their sizes relative to the granules vary significantly along the model sequence of stellar types.
\end{abstract}

Key words. stars: activity - stars: atmospheres - stars: low-mass - methods: numerical - magnetohydrodynamics (MHD) magnetic fields

\section{Introduction}

Cool main-sequence stars have outer convection zones (spectral types F to about M4) or are fully convective (spectral types later than M4). The turbulent flows in the electrically conducting plasma along with the stellar (differential) rotation support a hydromagnetic dynamo that constantly generates magnetic flux (see Charbonneau 2010, for a review). A fraction of this magnetic flux becomes buoyant and reaches the near-surface layers where it is continuously restructured by the convective flows (for reviews, see Fan 2009; Stein 2012; Schüssler 2013). At the same time, the magnetic field strongly affects the stellar near-surface layers. As the solar example illustrates, the magnetic field causes a variety of activity phenomena (see Schrijver \& Zwaan 2008, for a review). While many of these (e.g. flares and coronal mass ejections) are located in the higher atmospheric layers and in the interplanetary space, the solar magnetic field also has an impact on the photosphere and the convection zone. Sunspots, pores, and faculae are well-studied photospheric phenomena that are caused by strong $(1.5-3 \mathrm{kG})$ localised magnetic fields near the optical surface. Here, the magnetic field modifies the radiative and convective properties of the near-surface layers (see e.g. Rempel et al. 2009).

^ Movies associated to Fig. A.1 are available in electronic form at http://www . aanda.org
Like the Sun, many stars exhibit magnetic activity in the form of flares, X-ray emission, chromospheric emission lines, etc. (Schrijver \& Zwaan 2008). Lightcurve variability owing to spatial brightness variations (e.g. starspots) is nowadays detected in essentially every cool star (e.g. Reinhold et al. 2013). Measurements of activity indicators (such as X-ray luminosity or the $\mathrm{S}$-index) reveal that rapidly rotating (young) stars are much more magnetically active than the Sun (Reiners et al. 2014, and references therein). This also affects the detection of planetary companions around these stars (e.g. Jeffers et al. 2013) and probably also reduces the chance for habitability of the hosted planets (e.g. Vidotto et al. 2013).

For stars other than the Sun, current observations cannot directly spatially resolve activity-related phenomena in the photospheres. While most activity studies rely on activity indicators, for some stars it is possible to measure the surface magnetic field with spectroscopic and spectropolarimetric methods (see Reiners 2012, for a review). For cool main-sequence stars, these measurements yield global magnetic field strengths ranging from a few Gauss (from spectropolarimetry, e.g. Petit et al. 2008) up to $\gtrsim 3 \mathrm{kG}$ (Reiners \& Basri 2007). However, it is known from the Sun that the magnetic field at the surface is highly structured and inhomogeneous on length scales down to the current resolution limit of solar observations $(\sim 100 \mathrm{~km})$ and probably below. This local structure inevitably has a significant effect on spectroscopic and spectropolarimetric data (Stenflo 2013). In 
order to better understand the global magnetic field properties, knowledge of the local structure of the magnetised photosphere is therefore essential.

Since the 1990s, comprehensive 3D radiative magnetohydrodynamics (MHD) simulations have been used to simulate various magnetic phenomena in the solar near-surface layers, ranging from simple network and plage regions (Nordlund \& Stein 1990; Vögler et al. 2005) to pores (Cameron et al. 2007), sunspots (Rempel et al. 2009), active regions (Cheung et al. 2010), emergence of horizontal magnetic flux (Stein \& Nordlund 2006; Stein et al. 2011), and small-scale dynamo action (Vögler \& Schüssler 2007; Pietarila Graham et al. 2010). Until a few years ago, MHD simulations of this kind were only available for the Sun. Recently, the first comprehensive 3D radiative MHD simulations for stars other than the Sun were presented (Beeck et al. 2011; Wedemeyer et al. 2013; Steiner et al. 2013, 2014; Beeck et al. 2015b).

In Beeck et al. (2013a,b, hereafter Papers I and II), we discussed a sequence of non-magnetic (hydrodynamic) 3D simulations of near-surface convection with parameters close to mainsequence stars of spectral types ranging from F3 to M2. In Paper I, we analysed the overall structure of the convection and horizontally averaged stratifications. In Paper II, the granulation pattern in computed intensity images was studied and the effect of the 3D nature of the convection on limb darkening and on the profile of spectral lines was analysed. Here we continue this series of papers by considering the effects of a moderate magnetic field on the near-surface convection in cool main-sequence stars. For each set of stellar parameters presented in Paper I and Paper II, we consider three MHD simulation runs with a unipolar average magnetic field of various strengths. A brief description of the simulation setup is outlined in Sect. 2. In Sect. 3, the distribution of the magnetic field near the optical surface is discussed. In Sects. 4 and 5, we study the structure of small-scale magnetic flux concentrations. In the following sections, the effect of the magnetic field on the heating of the upper photosphere (Sect. 6) and on the convective flows (Sect. 7) is analysed. A brief conclusion follows in Sect. 8.

In a subsequent paper of the series (Beeck et al. 2015a, herafter Paper IV), we will consider the effects of the magnetic field on the limb darkening and on spectral lines.

\section{Simulation setup}

The simulations discussed in this paper have been obtained with the 3D radiative MHD code MURaM (Vögler 2003; Vögler et al. 2005). The version of the code used for the simulations considered here includes the modifications made by Rempel et al. (2009) to enhance the efficiency of the magnetic runs. We limit the Alfvén speed to $c_{\max }=60 \mathrm{~km} \mathrm{~s}^{-1}$ to prevent exceedingly small time steps. This only affects the nearly force-free regions near the tops of the simulations boxes and does not considerably influence the results discussed in this paper and in Paper IV. The numerical scheme is described in the appendix of Rempel et al. (2009)

The radiative transfer module of the code utilises the opacity binning method (see discussion in Paper I). For the nonmagnetic simulations considered here, opacity distribution functions provided by the ATLAS9 code (Kurucz 1993) were rearranged in four groups applying the $\tau$-sorting method (bin limits at $\log \tau_{\text {ref }}=0,-2,-4$; see discussion in Paper I). The magnetic simulations used the same binned opacities as the corresponding non-magnetic simulations.
Table 1. Stellar parameters and runtime (in simulated minutes) of the simulations.

\begin{tabular}{lrrrr}
\hline \hline Run $\left(\right.$ SpT- $\left.B_{0}{ }^{a}\right)$ & $\log g[\mathrm{cgs}]$ & $T_{\text {eff }}[\mathrm{K}]$ & $t_{1}{ }^{b}$ & $t_{\text {end }}{ }^{c}$ \\
\hline F3V-20G & 4.301 & $6885 \pm 6$ & 111 & 127 \\
F3V-100G & 4.301 & $6911 \pm 8$ & 106 & 121 \\
F3V-500G & 4.301 & $7003 \pm 5$ & 116 & 133 \\
\hline G2V-20G & 4.438 & $5779 \pm 9$ & 115 & 133 \\
G2V-100G & 4.438 & $5802 \pm 8$ & 108 & 126 \\
G2V-500G & 4.438 & $5864 \pm 9$ & 108 & 126 \\
\hline K0V-20G & 4.609 & $4858 \pm 2$ & 102 & 117 \\
K0V-100G & 4.609 & $4878 \pm 4$ & 78 & 91 \\
K0V-500G & 4.609 & $4901 \pm 2$ & 74 & 87 \\
\hline K5V-20G & 4.699 & $4376 \pm 2$ & 72 & 83 \\
K5V-100G & 4.699 & $4383 \pm 3$ & 61 & 73 \\
K5V-500G & 4.699 & $4402 \pm 2$ & 60 & 72 \\
\hline M0V-20G & 4.826 & $3907 \pm 1$ & 83 & 96 \\
M0V-100G & 4.826 & $3909 \pm 1$ & 58 & 69 \\
M0V-500G & 4.826 & $3906 \pm 1$ & 51 & 60 \\
\hline M2V-20G & 4.826 & $3691 \pm 1$ & 107 & 124 \\
M2V-100G & 4.826 & $3692 \pm 1$ & 59 & 68 \\
M2V-500G & 4.826 & $3679 \pm 1$ & 48 & 56 \\
\hline
\end{tabular}

Notes. ${ }^{(a)}$ SpT: spectral type (of corresponding non-magnetic run); $B_{0}$ : average vertical magnetic field strength. ${ }^{(b)} t_{1}$ : first time step considered in the analysis. ${ }^{(c)} t_{\text {end }}$ : current end time of simulation.

Six sets of stellar parameters ( $\log g$ and $\left.T_{\text {eff }}\right)$ roughly matching main-sequence stars of spectral types F3V, G2V, K0V, K5V, $\mathrm{M} 0 \mathrm{~V}$, and $\mathrm{M} 2 \mathrm{~V}$ were considered. The horizontal and vertical sizes of the computational domains were scaled with the expected granule sizes and pressure scale heights, respectively, to obtain similar setups for all sets of stellar parameters (for details, see Paper I). Snapshots of the non-magnetic simulations presented in Papers I and II were used as initial configuration for the mass density, the internal energy density, and the velocity field of the magnetic simulations. The initial magnetic field was unipolar, vertical with a uniform field strength of $B_{0}$. For each non-magnetic simulation, three simulation runs with $B_{0}=20 \mathrm{G}$, $100 \mathrm{G}$, and $500 \mathrm{G}$, respectively, and otherwise identical initial conditions were performed. In a transient restructuring phase, the flows advect the field into the converging downflow regions. After some time, the properties of magnetic field and flows become statistically stationary. The duration of the restructuring phase is of the order of the local convective turnover time, i.e. several minutes at the optical surface for all stellar parameters of our simulation sequence (and somewhat longer in the deeper layers). The simulations were run considerably longer to make sure that a statistically stationary state was reached. Table 1 gives the runtime of the simulation $t_{1}$ (in simulated stellar time) at which the first snapshots were considered and the total runtime, $t_{\mathrm{end}}$, of the simulations. The effective temperature, $T_{\text {eff }}$, of the simulations is regulated only by the entropy density of the inflowing plasma at the bottom of the computational domain. This quantity was fixed and identical in all four runs for each set of stellar parameters. Owing to the different (hot and cool) magnetic smallscale structures, there are slight differences in $T_{\text {eff }}$ between the different runs of the same star; values are given in Table 1 . Values of $T_{\text {eff }}$ of the non-magnetic runs are almost identical to the ones of the $20 \mathrm{G}$ runs and are given in Table 1 of Paper I. Overall the difference in $T_{\text {eff }}$ between runs of different $B_{0}$ is small. The reasons for the mostly positive deviations of the $100 \mathrm{G}$ and $500 \mathrm{G}$ runs from the non-magnetic and $20 \mathrm{G}$ runs will be discussed in Sect. 6. 

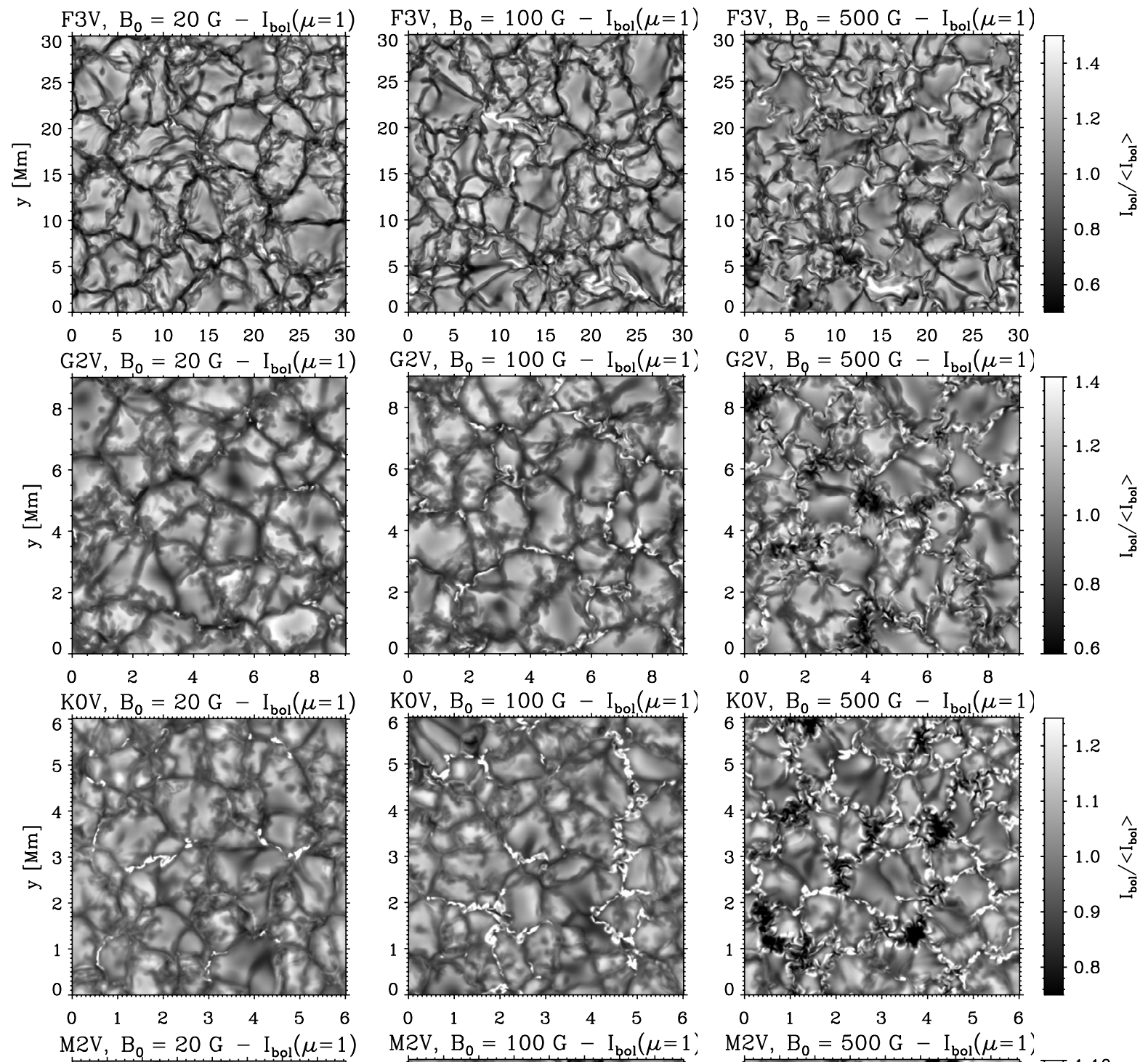

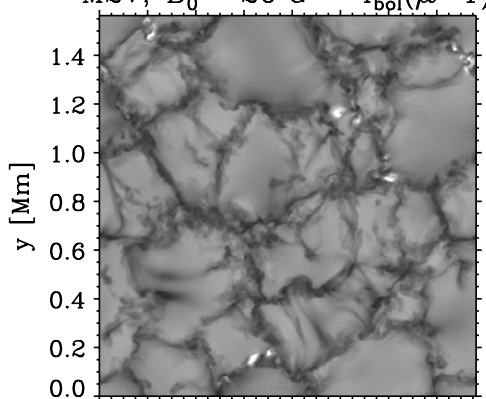

$\begin{array}{llllllllllllll}0.0 & 0.2 & 0.4 & 0.6 & 0.8 & 1.0 & 1.2 & 1.4\end{array}$ $\mathrm{x}[\mathrm{Mm}]$

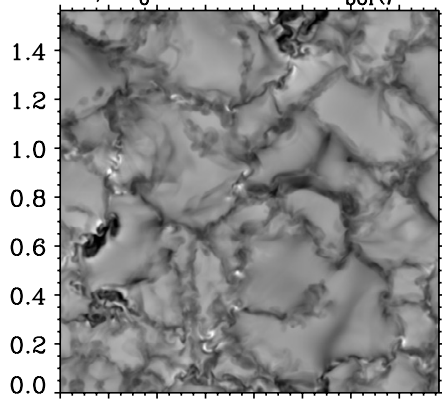

$\begin{array}{llllllllllll}0.0 & 0.2 & 0.4 & 0.6 & 0.8 & 1.0 & 1.2 & 1.4\end{array}$ $\mathrm{x}[\mathrm{Mm}]$

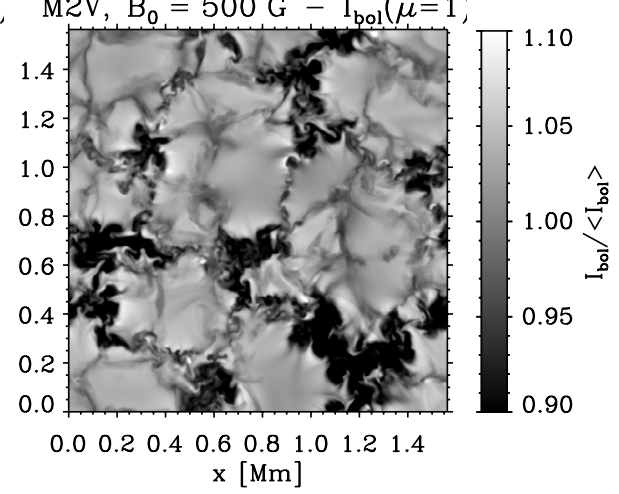

Fig. 1. Maps of the vertical bolometric intensity for twelve of the 18 magnetic simulation runs. For improved image contrast, the grey scale saturates at the values indicated by the colour scales on the right of each row.

The combination of three different values for $B_{0}$ with the six different sets of stellar parameters results in 18 magnetic simulations ${ }^{1}$. The six non-magnetic simulations presented in Paper I and Paper II serve as a reference, completing the grid of 24 simulation runs.

\footnotetext{
1 Movies of the vertical velocity and the magnetic field strength at the optical surface as well as the bolometric intensity of six of the 18 magnetic simulations are provided as online material (see Appendix A). Movies of all 18 magnetic simulations can be downloaded from http://www2 .mps .mpg . de/projects/solar-mhd/movies2015/
}

\section{Magnetic field distribution at the optical surface}

Figure 1 shows the vertically emerging bolometric intensity of all three magnetic runs for the four spectral types F3V, G2V, $\mathrm{K} 0 \mathrm{~V}$, and M2V. An analogous figure for the non-magnetic simulations is given in Fig. 2 of Paper I. In all simulations, regions of either strongly enhanced or significantly reduced intensity appear, mostly situated in the intergranular lanes. These regions coincide with the locations of strong magnetic flux concentrations (see Fig. 2). The relative number and size of dark and bright 

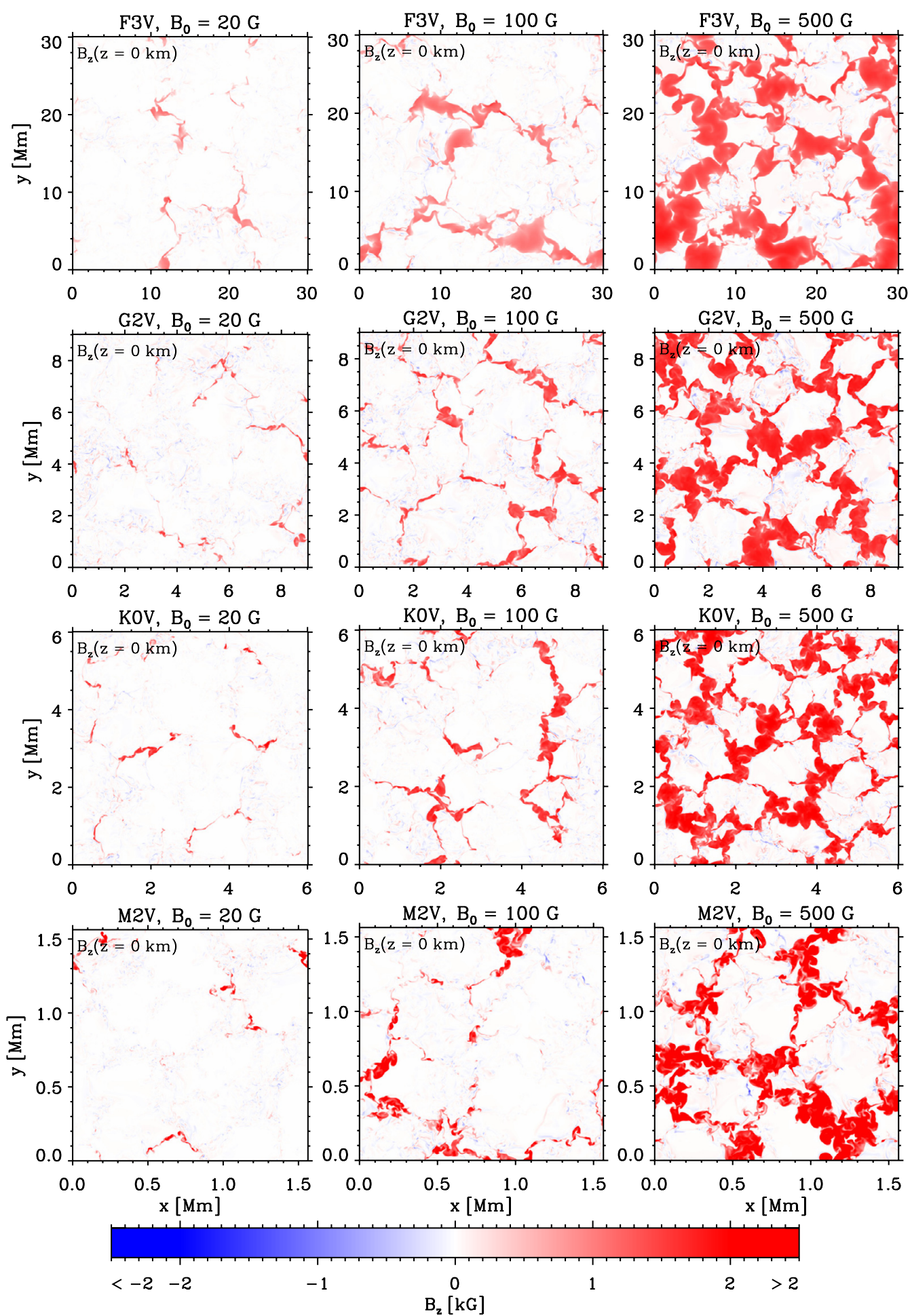

Fig. 2. Maps of the vertical component, $B_{z}$, of the magnetic field at $z=0$, the average geometrical depth of the optical surface (same simulation runs and at the same time step as in Fig. 1).

regions as well as their intensity contrast strongly depend on the spectral type and the amount of magnetic flux in the simulation box (i.e. on $B_{0}$ ). This is discussed in some more detail in Sect. 4. The most striking difference appears between the M-star simulations on the one hand and all other simulations on the other: in the F-, G- and K-stars simulations, only for $B_{0}=500 \mathrm{G}$ a few dark features appear among the mostly bright magnetic structures; in contrast, in the M2V star (and in the M0V star; not shown here), dark magnetic structures frequently form already in the $100 \mathrm{G}$ runs and the intensity maps of the $500 \mathrm{G}$ runs are dominated by large dark structures while there are essentially no bright magnetic structures left (cf. Beeck et al. 2011).

Figure 2 shows maps of the vertical component $B_{z}$ of the magnetic field at $z=0$, the mean height level of the optical surface for the same selection of simulation runs and at the same time steps as Fig. 1. The strong concentration of the magnetic flux in some of the convective downflows leads to local field strengths of up to several $\mathrm{kG}$ in all stars, even in the case of $B_{0}=20 \mathrm{G}$. The maximum of the field strength at the optical surface depends only weakly on $B_{0}$, especially in the cooler stars 
B. Beeck et al.: 3D simulations of near-surface convection in main-sequence stars. III.
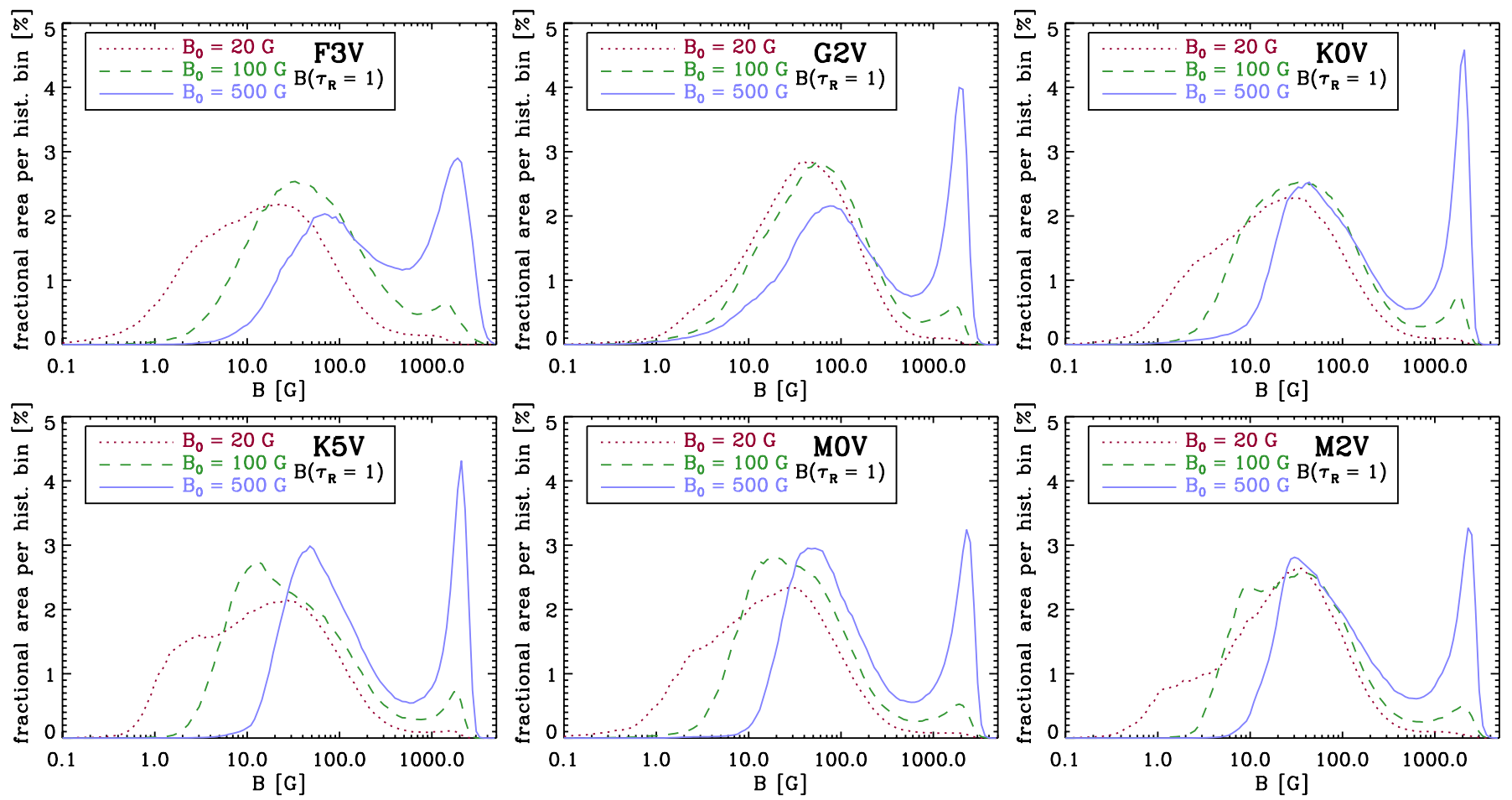

Fig. 3. Histograms of the modulus of the magnetic field strength, $B$, at the optical surface for all 18 magnetic simulation runs (the histogram bin size is $0.04 \mathrm{dex}$ ).

Table 2. Properties of the magnetic field at the $\tau_{\mathrm{R}}=1$ surface.

\begin{tabular}{crlcc}
\hline \hline SpT $^{a}$ & $\left\langle B_{z}\right\rangle$ & $\operatorname{Max}\left(B\left(\tau_{\mathrm{R}}=1\right)\right)$ & $\sqrt{\left\langle B^{2}\right\rangle}$ & $B_{\text {strong }}{ }^{b}$ \\
\hline F3V & 20 & $2040 \pm 160$ & $150 \pm 4$ & - \\
F3V & 100 & $4250 \pm 550$ & $445 \pm 4$ & $1418 \pm 67$ \\
F3V & 500 & $5180 \pm 510$ & $1160 \pm 10$ & $1886 \pm 59$ \\
\hline G2V & 20 & $2310 \pm 110$ & $178 \pm 6$ & - \\
G2V & 100 & $3250 \pm 410$ & $412 \pm 10$ & $1702 \pm 74$ \\
G2V & 500 & $3550 \pm 150$ & $1022 \pm 11$ & $1990 \pm 18$ \\
\hline K0V & 20 & $2890 \pm 160$ & $175 \pm 7$ & - \\
K0V & 100 & $2930 \pm 130$ & $425 \pm 3$ & $1824 \pm 24$ \\
K0V & 500 & $3390 \pm 140$ & $1031 \pm 6$ & $2056 \pm 11$ \\
\hline K5V & 20 & $2640 \pm 170$ & $176 \pm 4$ & - \\
K5V & 100 & $2980 \pm 80$ & $418 \pm 6$ & $1823 \pm 48$ \\
K5V & 500 & $3550 \pm 140$ & $1043 \pm 3$ & $2099 \pm 13$ \\
\hline M0V & 20 & $3400 \pm 240$ & $185 \pm 5$ & - \\
M0V & 100 & $3900 \pm 200$ & $436 \pm 4$ & $1917 \pm 41$ \\
M0V & 500 & $4200 \pm 200$ & $1075 \pm 3$ & $2326 \pm 15$ \\
\hline M2V & 20 & $3500 \pm 580$ & $182 \pm 3$ & - \\
M2V & 100 & $4090 \pm 370$ & $440 \pm 8$ & $1994 \pm 85$ \\
M2V & 500 & $4450 \pm 320$ & $1067 \pm 7$ & $2352 \pm 57$ \\
\hline
\end{tabular}

Notes. All values in G; errors give temporal $1 \sigma$ scatter. ${ }^{(a)}$ Spectral type of the simulation; ${ }^{(b)}$ peaks of the histograms of Fig. 3.

of the model sequence: for instance, in the M2V star, it is $3.5 \mathrm{kG}$ in the $20 \mathrm{G}$ run and $4.5 \mathrm{kG}$ in the $500 \mathrm{G}$ run (more values given in Table 2).

As the typical field strength in the magnetic flux concentrations does not strongly vary with $B_{0}$, the area fraction of these structures increases with increasing $B_{0}$. This is also illustrated by Fig. 3, which gives the histograms of the magnetic field modulus, $B\left(\tau_{\mathrm{R}}=1\right)$, at the optical surface. In the $500 \mathrm{G}$ and $100 \mathrm{G}$ runs, these histograms have two peaks (using logarithmically equidistant bins), one at relatively low values between 10 and $100 \mathrm{G}$ (in most simulations, this peak falls between 30 and $50 \mathrm{G}$ ) and one at a high field strength of about $2 \mathrm{kG}$. The latter peak represents the typical field strength at the optical surface in the magnetic flux concentrations and is denoted as $B_{\text {strong }}$ in what follows. Values for $B_{\text {strong }}$ obtained from a Gaussian fit to the histogram peak range between $1.4 \mathrm{kG}$ and $2.4 \mathrm{kG}$ (see Table 2). $B_{\text {strong slightly }}$ increases with $B_{0}$ and towards the cooler end of our sequence. In the $20 \mathrm{G}$ runs there is no strong-field peak, but the distributions of $B\left(\tau_{\mathrm{R}}=1\right)$ still show an extended tail towards high values of the field strength.

The weak dependence of $B_{\text {strong }}$ on $B_{0}$ is consistent with the rms values of $B$, which are largely independent of stellar parameters. This supports a simple two-component model of the surface field: if one assumes a fraction $f$ of the stellar surface to have a field strength $B_{\text {strong }}$ and the remaining fraction $1-f$ to have a field strength $B_{\text {weak }} \approx 0$ and further assumes the field to be mostly vertical and unipolar, one obtains $f=B_{0} / B_{\text {strong }}$, from which for the rms $\left\langle B^{2}\right\rangle^{1 / 2}=\left(f B_{\text {strong }}^{2}\right)^{1 / 2}=\left(B_{0} B_{\text {strong }}\right)^{1 / 2}$. For $B_{\text {strong }}=2 \mathrm{kG}$ one obtains an rms magnetic field of 200,447 , and $1000 \mathrm{G}$ for $B_{0}=20,100$, and $500 \mathrm{G}$, respectively, which is very close to the values actually found in the simulations (see Table 2). Owing to the strong height dependence of the magnetic field (cf. Fig. 4), as well as to the local impact of the magnetic field on the atmosphere structure, a two-component model appears nonetheless inadequate to compute profiles of magnetically sensitive spectral lines (cf. Paper IV).

\section{Structure of magnetic flux concentrations}

Figure 4 shows vertical cuts through one of the magnetic flux concentrations for each of the $100 \mathrm{G}$ runs. The light-blue dashed curves give iso-contours of the magnetic field strength of 500,1000 , and $1500 \mathrm{G}$, which indicate the locations of strong magnetic flux concentrations. In the flux concentrations, the magnetic pressure $p_{\text {mag }}=B^{2} /(8 \pi)$ becomes substantial. As the atmospheres are roughly in hydrostatic equilibrium, the gas pressure and the (mass) density are locally reduced in regions of strong magnetic field. The underlying grey-scale image gives the density distribution and confirms that the flux concentrations 

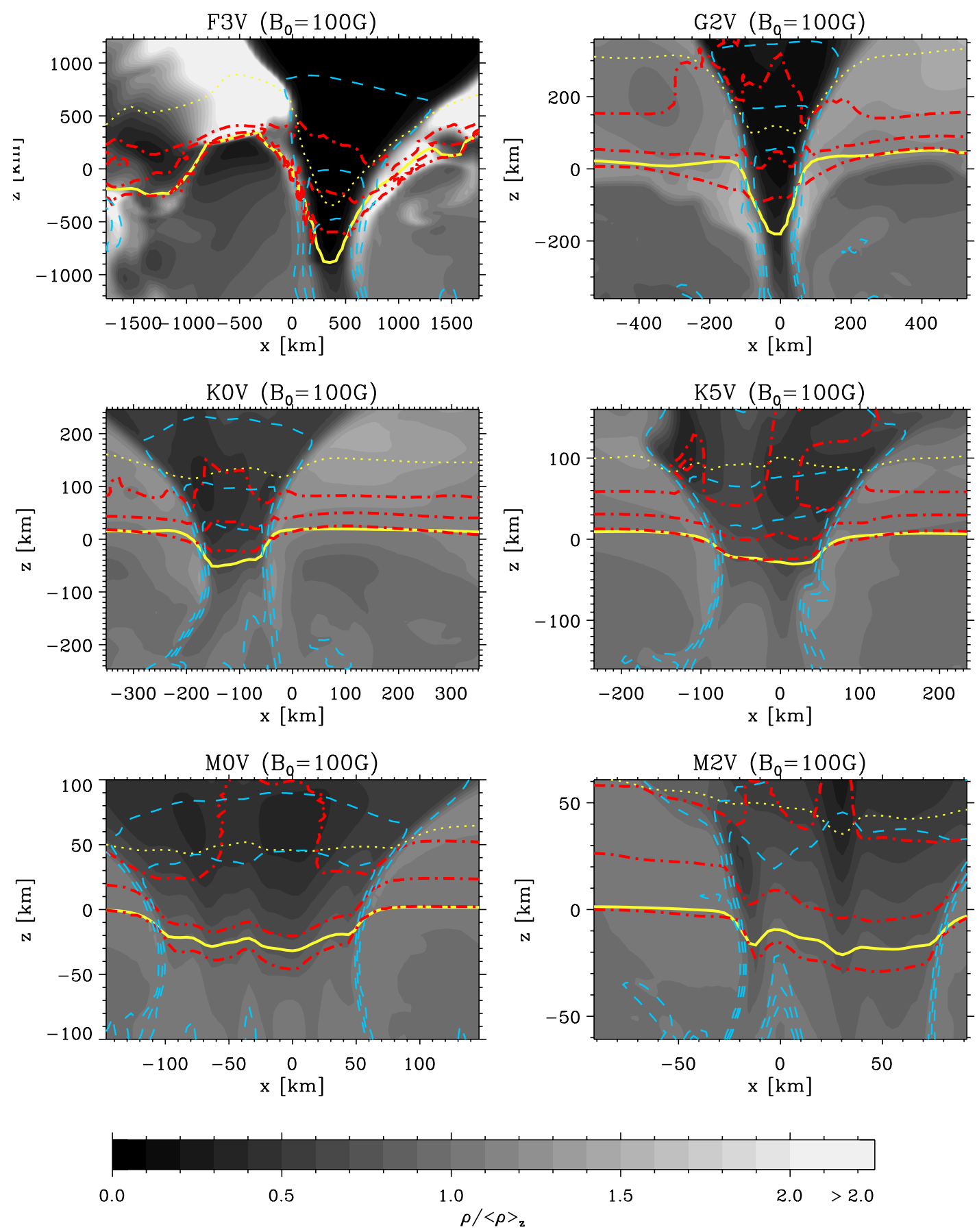

Fig. 4. Vertical cuts through typical magnetic flux concentrations in the six simulations with $B_{0}=100 \mathrm{G}$. The grey scale indicates the density $\varrho /\langle\varrho\rangle_{z}$ relative to the horizontal mean density; the solid (dotted) yellow curve represents the $\tau_{\mathrm{R}}=1\left(\tau_{\mathrm{R}}=0.01\right)$ surface; the dashed blue contours indicate iso- $B$ contours of 500,1000 , and $1500 \mathrm{G}$; the red dash-dotted curves indicate the $T / T_{\text {eff }}=0.9,1.0$, and 1.1 surfaces. $x$ and $z$ coordinates are to scale for each panel but differ by more than one order of magnitude for the different stellar models.

are all under-dense with respect to the horizontal (iso- $z$ ) average stratification. However, the degree of evacuation strongly varies throughout the model sequence. The hottest two stars (F3V and $\mathrm{G} 2 \mathrm{~V}$ ) show the strongest evacuation in the upper (optically thin) part of the magnetic structures, with a density of the order of only $10 \%$ of its horizontal mean value (in the first pressure scale height above the optical surface). In the K-dwarf simulations, the density in the magnetic structures reaches still about $30-50 \%$ of the horizontal average density and in the M-dwarf simulations, where evacuation is more confined to the upper layers, there is almost no evacuation near the optical surface. This effect is probably a result of the much lower superadiabaticity in the cooler stars (see Paper I), which makes the convective collapse less efficient (cf. Rajaguru et al. 2002). The reduced efficiency of the convective collapse results in reduced ratio between the magnetic pressure and the gas pressure (see Sect. 5).

The solid yellow contours in Fig. 4 outline the $\tau_{R}=1$ surface (=optical surface). In all simulations, magnetic flux concentrations cause a local depression in the optical surface, which is referred to as Wilson depression in analogy to the same phenomenon in sunspots. The depression is caused by the partial evacuation in the near-surface layers, which results in a lower absorption coefficient $\kappa \varrho$ (and thus longer mean free path length of the photons). 
B. Beeck et al.: 3D simulations of near-surface convection in main-sequence stars. III.
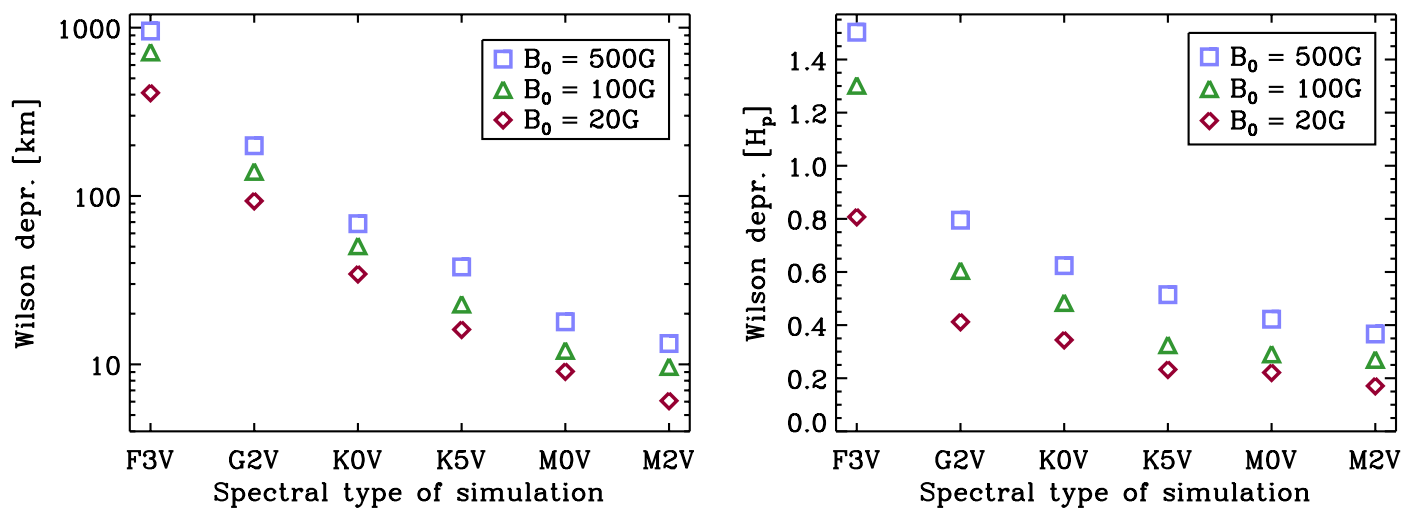

Fig. 5. Wilson depressions. Left panel: difference in average geometrical depth of the $\tau_{\mathrm{R}}=1$ surface between magnetic $\left(B\left(\tau_{\mathrm{R}}=1\right)>750 \mathrm{G}\right)$ and non-magnetic area $\left(B\left(\tau_{\mathrm{R}}=1\right)<750 \mathrm{G}\right)$ as a measure for the depth of the Wilson depressions. Right panel: downward shift of the optical surface within the magnetic flux concentrations in terms of pressure scale height (measured outside the flux concentrations).

The red dash-dotted curves outline iso- $T$ contours of $0.9,1.0$ and 1.1 times the effective temperature. In all cases, the $1.1 T_{\text {eff }}$ iso-contour has a lower geometrical height within the magnetic flux concentrations than outside: at the same geometrical depth, all flux concentrations are cool around $z=0$. However, the optical surface depressions are in most cases larger than the depressions of the iso- $T$ contours: at the same optical depth, most flux concentrations shown here are hotter than the surroundings (with the notable exception of the M-star simulations). The contours of 1.0 and $0.9 T_{\text {eff }}$ are mostly above the optical surface and show a more complicated structure.

The optically thin plasma in the photospheric layers of the flux concentrations is heated by various mechanisms including adiabatic heating in downflows (subadiabatic temperature gradient), radiative heating, as well as viscous and Ohmic dissipation (see Sect. 6). In the layers directly above the optical surface, the absorption coefficient is still high enough for radiative heating to be important. This leads to heating near inclined and concavely curved parts of the optical surface (side walls of the depressions). As a consequence, magnetic flux concentrations with sizes comparable to the Wilson depression have higher temperatures than the non-magnetic surroundings near the optical surface. In flux concentrations with a large diameter (compared to their depth), this heating is only effective near the side walls. This explains why in the F-, G-, and K-type simulations the larger magnetic flux concentrations become dark in their centres while smaller ones are bright. In the M-star simulations, the depressions in the optical surface are all very shallow owing to the low degree of evacuation in the upper part of the flux concentration. Consequently, even a moderate amount of magnetic flux generates a structure with a large radius compared to its Wilson depression. Therefore, many magnetic flux concentrations on $\mathrm{M}$ stars are dark.

The dotted yellow contours in Fig. 4 give the $\tau_{\mathrm{R}}=0.01$ surface. Many photospheric spectral lines (such as the ones discussed in PaperS II and IV) are most sensitive to the conditions around this optical depth. The magnetic field strength in the flux concentrations and their filling factor may differ by a significant amount between the optical surface and this higher iso- $\tau_{\mathrm{R}}$ surface. Note that the depressions in the $\tau_{\mathrm{R}}=0.01$ surface are generally shallower than the depressions of the optical surface. This can partly be attributed to the heating mechanisms, which lead to a positive temperature deviation (with respect to the horizontal average) at this height (and above) and thus to a higher opacity, partially compensating the diminishing effect of the reduced density on the absorption coefficient.
Figure 5 shows the dependence of the depth Wilson depression on the spectral type of the simulation and on $B_{0}$. In the left panel of the figure, the depth is given in terms of $\mathrm{km}$. By far the deepest Wilson depressions are observed in the F3V star (about $950 \mathrm{~km}$ in the $500 \mathrm{G}$ run). The depth of the depressions decreases strongly along our simulation sequence towards cooler stars as well as with decreasing $B_{0}$. The dependence on $B_{0}$ is mainly caused by the different size of the structures: in most cases, the depressions are deeper in their central part than near their boundaries (where the field strength is somewhat lower and where the side-wall heating can result in a significant increase in temperature and thus opacity). As the flux concentrations become larger at higher values of $B_{0}$, their area becomes, on average, larger in relation to their perimeter. Therefore, these "boundary" effects become less important at larger $B_{0}$, resulting in a greater average depth of the Wilson depressions. In the right panel of Fig. 5, the depth of the Wilson depressions is given in terms of pressure scale height. Although the pressure scale height decreases by more than one order of magnitude (from $\sim 500 \mathrm{~km}$ in F3V to $\sim 35 \mathrm{~km}$ in M2V, see Paper I), the decreasing trend of the depth of the depressions along the model sequence still remains. The depth of the depressions ranges from $0.17 H_{\mathrm{p}}(\mathrm{M} 2 \mathrm{~V}$, $\left.B_{0}=20 \mathrm{G}\right)$ to $1.5 H_{\mathrm{p}}\left(\mathrm{F} 3 \mathrm{~V}, B_{0}=500 \mathrm{G}\right)$. Here, a threshold of $B\left(\tau_{\mathrm{R}}=1\right)=750 \mathrm{G}$ was applied to identify magnetic flux concentrations. Using different threshold values does not influence the results qualitatively, but has a quantitative impact, in particular for the $20 \mathrm{G}$ and $100 \mathrm{G}$ runs: with a threshold of $500 \mathrm{G}$ rather than $750 \mathrm{G}$, the depth of the Wilson depressions decreases by roughly $20 \%, 15 \%$, and $5 \%$ for the $20 \mathrm{G}, 100 \mathrm{G}$ and $500 \mathrm{G}$ runs, respectively. With a threshold of $1000 \mathrm{G}$ rather than $750 \mathrm{G}$, the depth of the Wilson depression increases by roughly $15 \%, 10 \%$, and $5 \%$ for the $20 \mathrm{G}, 100 \mathrm{G}$ and $500 \mathrm{G}$ runs, respectively. The small impact on the $500 \mathrm{G}$ runs confirms that the dependence on $B_{0}$ is mostly due to the boundary effects discussed above.

\section{Balance of magnetic, thermal and turbulent pressures}

Owing to the Wilson depressions, the optical surface within the magnetic flux concentrations is below its average level $z=0$. As the magnetic field lines fan out in the upper parts of the flux concentrations, the field strength is higher at the optical surface (where it defines $B_{\text {strong }}$ ) than at $z=0$ (cf. Fig. 4). The magnetic field strength $\langle B(z=0)\rangle_{\text {int }}$ in the magnetic flux concentrations varies somewhat more strongly along our sequence of spectral 

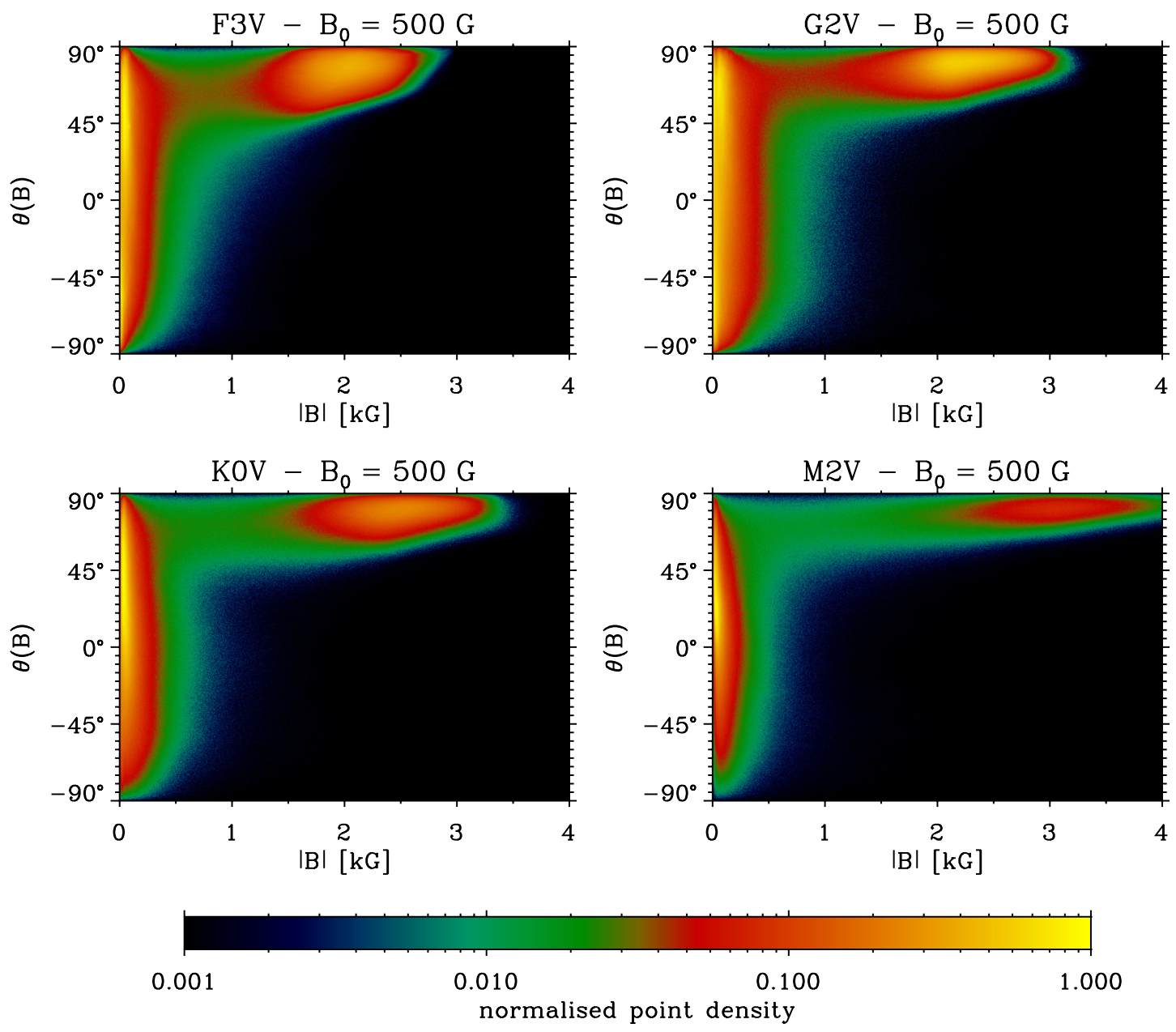

Fig. 6. $2 \mathrm{D}$ histograms of the modulus of the field strength versus the inclination of the field $\left(0^{\circ}=\right.$ horizontal; $\pm 90^{\circ}=$ vertical $)$ for the $500 \mathrm{G}$ runs of the F3V, G2V, K0V and M2V stars.

types than $B_{\text {strong, }}$ but still much less than one would expect if the convective collapse for thin flux tubes (cf. Spruit 1976) were equally efficient for different stellar parameters: this would yield $\left\langle B^{2}(z=0)\right\rangle_{\text {int }} /(8 \pi)=p_{\text {mag,int }}(z=0) \propto p_{\text {gas,ext }}(z=0)$, i.e. the magnetic pressure inside the flux tubes (referred to as "internal" in what follows) should roughly scale with the thermal pressure outside of the flux tubes (referred to as "external" in what follows), as the end products of an efficient convective collapse are strongly evacuated magnetic structures which are roughly in pressure equilibrium with the external gas pressure. Table 3 lists horizontal averages (at $z=0$ ) of the internal magnetic pressure, the external gas pressure, and the external "turbulent pressure" (twice the kinetic energy density) for the $500 \mathrm{G}$ runs. Magnetic flux concentrations were defined as the area where $B(z=0)>750 \mathrm{G}$. The internal magnetic pressure in the $\mathrm{K}$ - and particularly in the M-star simulations does not reach equipartition with the external gas pressure, as it approximately does for the Sun and the F3V star. In our model sequence (which roughly follows the main sequence through the $T_{\text {eff }}-\log g$ plane), the decreasing efficiency of the convective collapse towards cooler stars is roughly compensated by the increasing external gas pressure so that $B_{\text {strong }}$ becomes almost independent of the stellar type in our sequence.

Figure 6 shows 2D histograms of the magnetic field modulus versus the inclination, $\theta$, of the field with respect to the surface $\left( \pm 90^{\circ}=\right.$ vertical; $0^{\circ}=$ horizontal $)$ in a layer covering half a pressure scale height both below and above $z=0$. The
Table 3. Magnetic pressure (in the flux concentrations) and external turbulent and thermal gas pressure at $z=0$ in the $500 \mathrm{G}$ runs in dyn $\mathrm{cm}^{-2}$.

\begin{tabular}{cccc}
\hline \hline Simulation & $p_{\text {mag }}$ & $\rho v^{2}$ & $p_{\text {gas }}$ \\
\hline F3V & $7.41 \times 10^{4}$ & $3.21 \times 10^{4}$ & $7.65 \times 10^{4}$ \\
G2V & $9.39 \times 10^{4}$ & $3.87 \times 10^{4}$ & $1.46 \times 10^{5}$ \\
K0V & $1.10 \times 10^{5}$ & $3.31 \times 10^{4}$ & $2.28 \times 10^{5}$ \\
K5V & $1.23 \times 10^{5}$ & $3.49 \times 10^{4}$ & $3.15 \times 10^{5}$ \\
M0V & $1.47 \times 10^{5}$ & $2.76 \times 10^{4}$ & $5.11 \times 10^{5}$ \\
M2V & $1.51 \times 10^{5}$ & $2.00 \times 10^{4}$ & $5.99 \times 10^{5}$ \\
\hline
\end{tabular}

magnetic field in the near-surface layers falls into two components (cf. Fig. 3): in regions with high field strength of a few $\mathrm{kG}$ (i.e. $\sim B_{\text {strong }}$ ) the field is mostly close to vertical while weakfield regions show almost isotropic field inclinations with a weak tendency towards positive inclinations (i.e. the field direction of the dominant polarity). There is a dependence on spectral type: in the F3V-star simulation, the inclinations in the strong-field regime are less concentrated towards the vertical (with inclinations down to about $45^{\circ}$ ), while the field inclination in the $\mathrm{M} 2 \mathrm{~V}$ star is much closer to vertical (always above $60^{\circ}$ ) in this regime. In the latter simulation, the separation between weak and strong field is also more pronounced. Along the model sequence there is a smooth transition from the F-star-like to the M-star-like distribution as illustrated by the other two spectral types shown in Fig. 6. This is related to the different balance of magnetic and 
B. Beeck et al.: 3D simulations of near-surface convection in main-sequence stars. III.
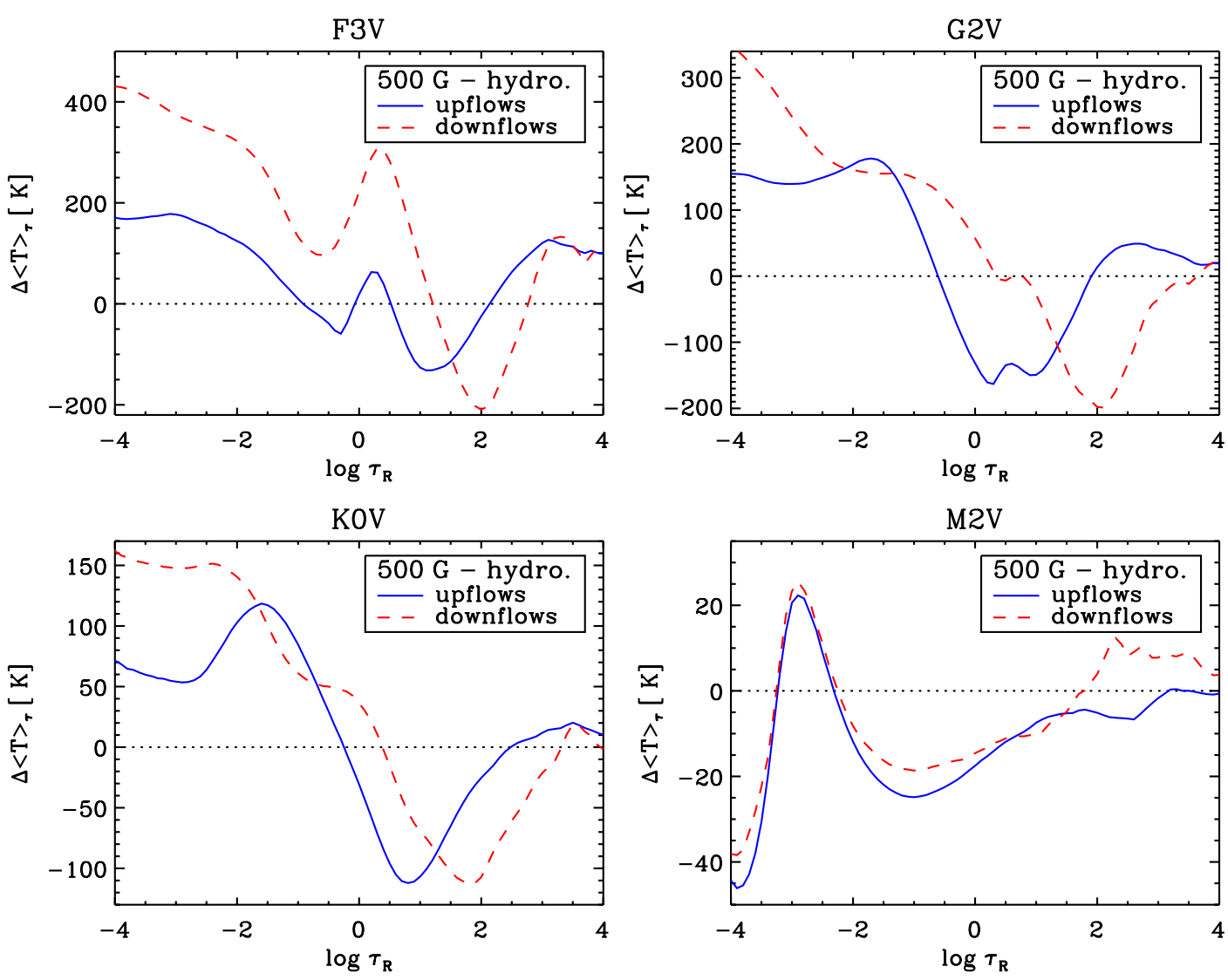

Fig. 7. Average temperature difference between the $500 \mathrm{G}$ and non-magnetic runs in up- and downflows for the F3V, G2V, K0V, and $\mathrm{M} 2 \mathrm{~V}$ simulations.

turbulent pressures (see Table 3): in the F3V star, the ratio between internal magnetic pressure and external turbulent pressure is about 2.5 whereas it is 30 in the M2V star. This renders the magnetic flux concentrations in the F3V star much more susceptible to the surrounding plasma motions, leading to a larger spread in field strength and orientation. In contrast, the magnetic flux concentrations in the M-star simulations are quite stationary and less strongly influenced by the surrounding convection.

\section{Heating of the upper photosphere}

Figure 7 gives the difference in $\langle T\rangle_{\tau}\left(\tau_{\mathrm{R}}\right)$, the temperature averaged over iso- $\tau_{\mathrm{R}}$ surfaces, between $500 \mathrm{G}$ runs and non-magnetic runs, shown separately for up- and downflows. The local modification of the temperature in the flux concentrations shows in the average temperature profiles $\langle T\rangle_{\tau}\left(\tau_{\mathrm{R}}\right)$. Interestingly, the impact of the magnetic field on the temperature structure is qualitatively and quantitatively similar in up- and downflows, although the magnetic flux concentrations are mostly located in downflows: the temperature is somewhat increased above the optical surface and decreased below the optical surface with the exception of the M stars, where the impact is smaller in amplitude and more complicated. The reduced temperature directly below the surface and the enhanced temperature above the surface imply a reduced average temperature gradient in the near-surface layers. This is a consequence of the somewhat more efficient radiative energy transport across the optical surface. Owing to the local depressions in the optical surface, the area of the radiating surface is increased. As a consequence, the radiative flux and hence the effective temperature of the atmospheres is somewhat higher in the magnetic runs. As the M stars only have very shallow Wilson depressions, they do not show a decreased temperature gradient around $\log \tau_{\mathrm{R}}=0$ in the magnetic runs.
In most cases, the middle and upper photosphere $\left(\log \tau_{\mathrm{R}} \lesssim\right.$ $-2)$ is warmer in the magnetic runs than in the non-magnetic runs. While the heating of the lower photosphere above magnetic flux concentrations is mainly due to radiation, the enhanced heating of the upper photosphere is caused by enhanced viscous dissipation and Ohmic heating in regions of substantial magnetic field. In the upper photosphere, the magnetic pressure dominates the thermal pressure. The magnetic field couples the plasma at this height to the magnetic flux concentrations below, which are subject to the interaction with the convective flows. This excites magneto-acoustic waves as well as torsional motions, which are then dissipated at greater heights. For solar simulations this effect has been demonstrated by Moll et al. (2012) and Shelyag et al. (2013). Observational evidence for magnetoacoustic waves in magnetic bright points in the Sun has been given by Jafarzadeh et al. (2013).

Figures 8 and 9 illustrate the heating of the upper photosphere. The figures represent horizontal cuts through the G2V-, K0V- and M2V-star simulations at a height of 4.6 pressure scale heights above the mean level of the optical surface. Figure 8 gives the non-magnetic runs of these stars, while Fig. 9 shows the $100 \mathrm{G}$ runs. The upper row of plots illustrates the vertical velocity at that height. In the non-magnetic case (Fig. 8), the velocity amplitude is relatively low and the horizontal scale of the structures is comparable to the granule size. In the $100 \mathrm{G}$ runs, small-scale structures with high vertical velocities appear, which are associated with the magnetic structures (bottom row of Fig. 9). The second row of the figures shows the modulus of the horizontal velocity at the same height.In the non-magnetic solar case, we find regions of high horizontal velocity that are usually accompanied by a shock front at an interface between up- and downflows. In the cooler stars, the horizontal velocities 

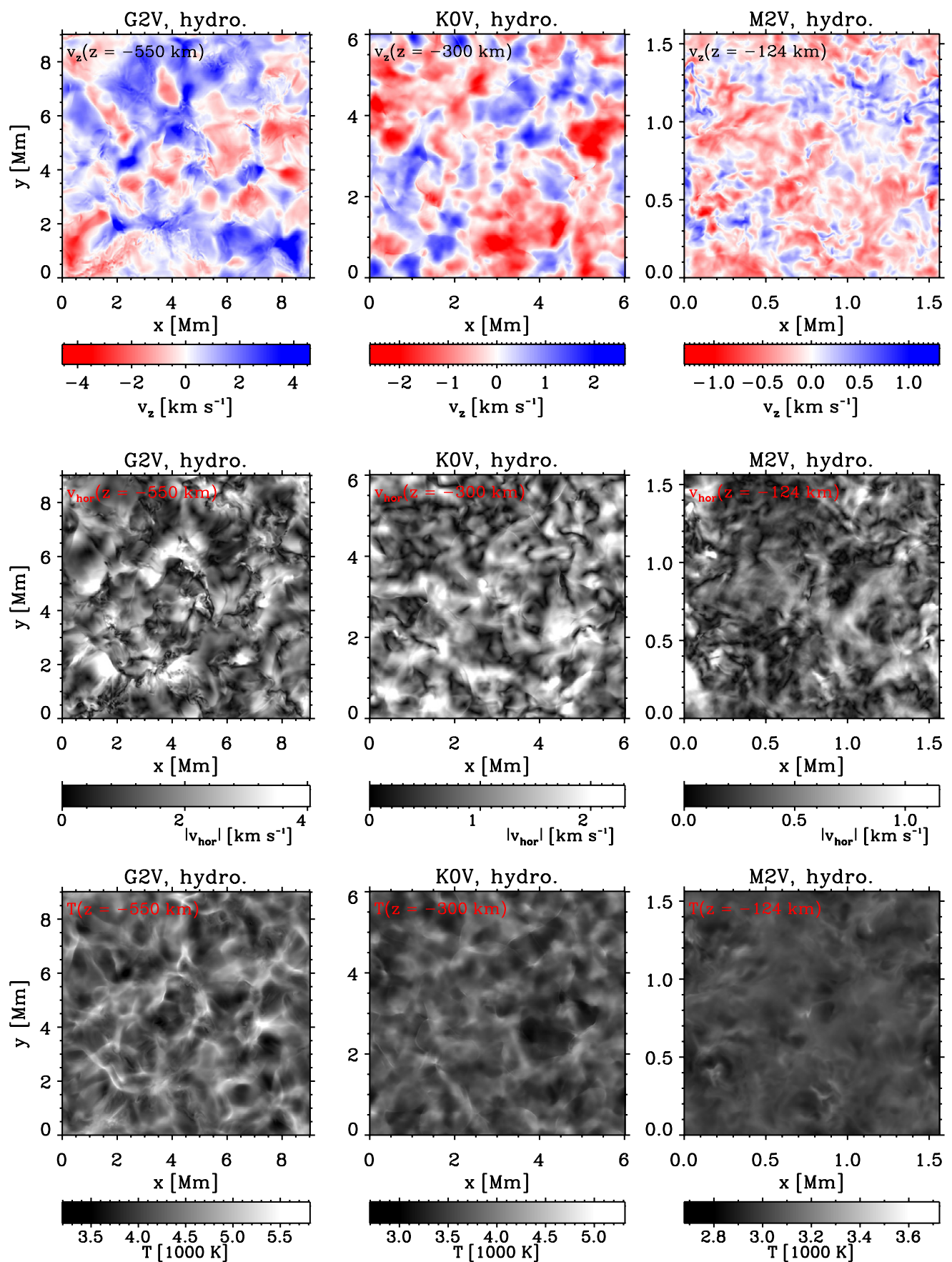

Fig. 8. Horizontal cuts through the boxes of the G2V, K0V, and M2V simulations (from left to right) without magnetic field. All cuts are situated such that $\langle p\rangle_{z}=0.01 p_{0}$, i.e. about 4.6 pressure scale heights above the optical surface; the geometrical depth of this level is given in each panel. From top to bottom: vertical velocity, $v_{z}$, horizontal speed, $\left|v_{\text {hor }}\right|:=\left(v_{x}^{2}+v_{y}^{2}\right)^{1 / 2}$, and temperature, $T$ are given; see also Fig. 9 .

are relatively low and no shocks appear. In the $100 \mathrm{G}$ run, the differences between spectral types almost dissappear as all simulations show high-velocity horizontal flows with small horizontal length scale at the same places where the small-scale vertical velocity structures appear. The third row of the figures shows the temperature at the same height. In the non-magnetic solar simulation, the shocks are visible in the temperature as the localised viscous heating produces a high temperature at the shock fronts. In the non-magnetic runs of the cooler stars, the temperature fluctuates less at this level. In the magnetic simulations, the small-scale high-velocity structures correspond to regions of high temperature fluctuations (and in most cases regions of higher temperature). There are some localised maxima where the temperature is more than $1000 \mathrm{~K}$ (in the solar case even more than $2500 \mathrm{~K}$ ) above the horizontal mean value. In Fig. 9, the fourth row shows the magnetic field strength at this height. The temperature maxima are all located in regions with high magnetic field strength and often a significant gradient of the magnetic field. Although the general structure of the magnetic field is much smoother on the larger scales in this height, there is significant fine structure (e.g. current sheets and vortices), which is responsible for the strong heating on small scales.

\section{Impact on convective flows}

As described in Sect. 3, the velocity field is responsible for the very inhomogeneous structure of the magnetic field in the first 
B. Beeck et al.: 3D simulations of near-surface convection in main-sequence stars. III.
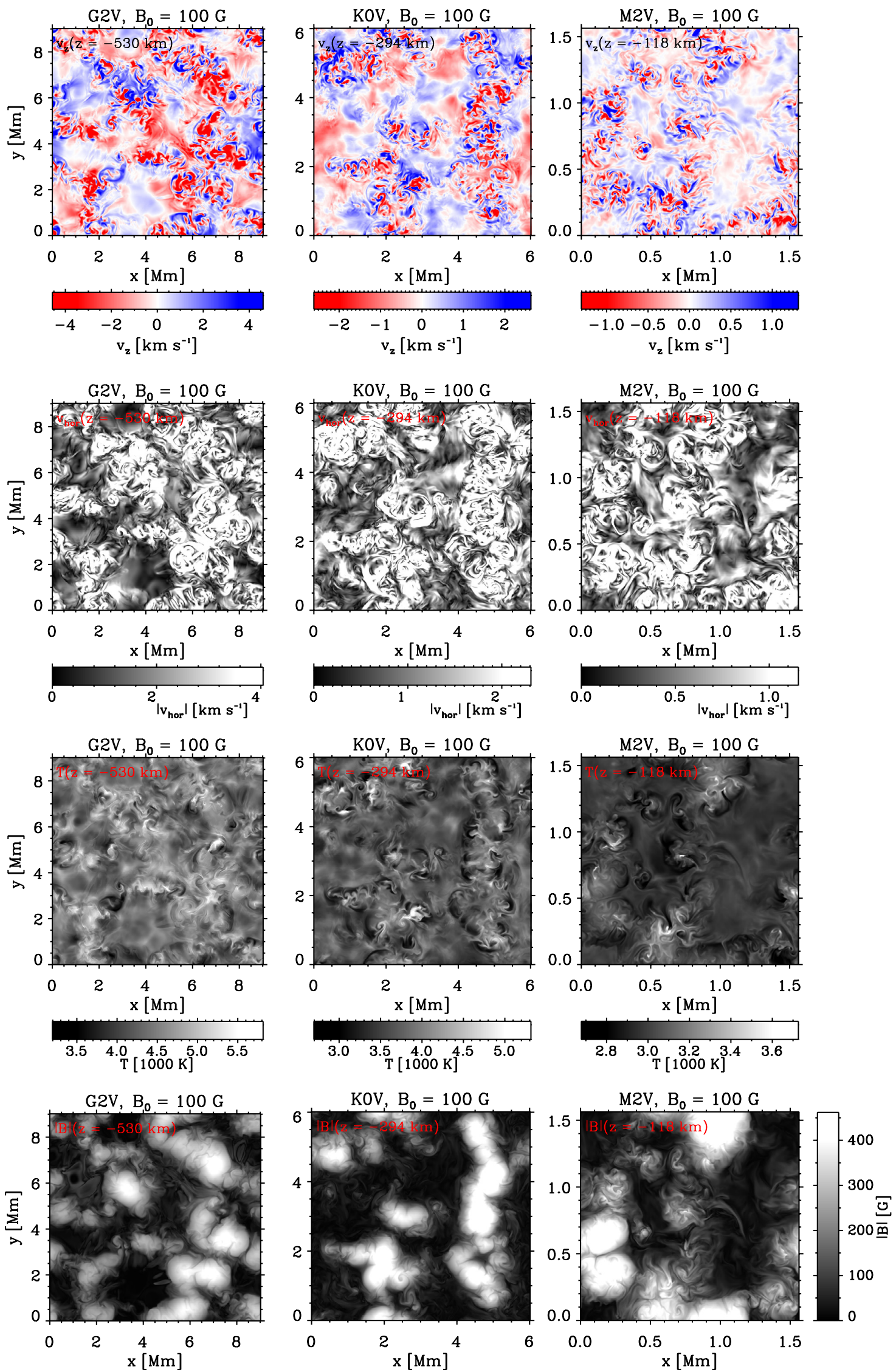

Fig. 9. Upper three rows: same quantities as Fig. 8, but for the $100 \mathrm{G}$ runs of the respective stellar model. Bottom row: horizontal field at the same height. 

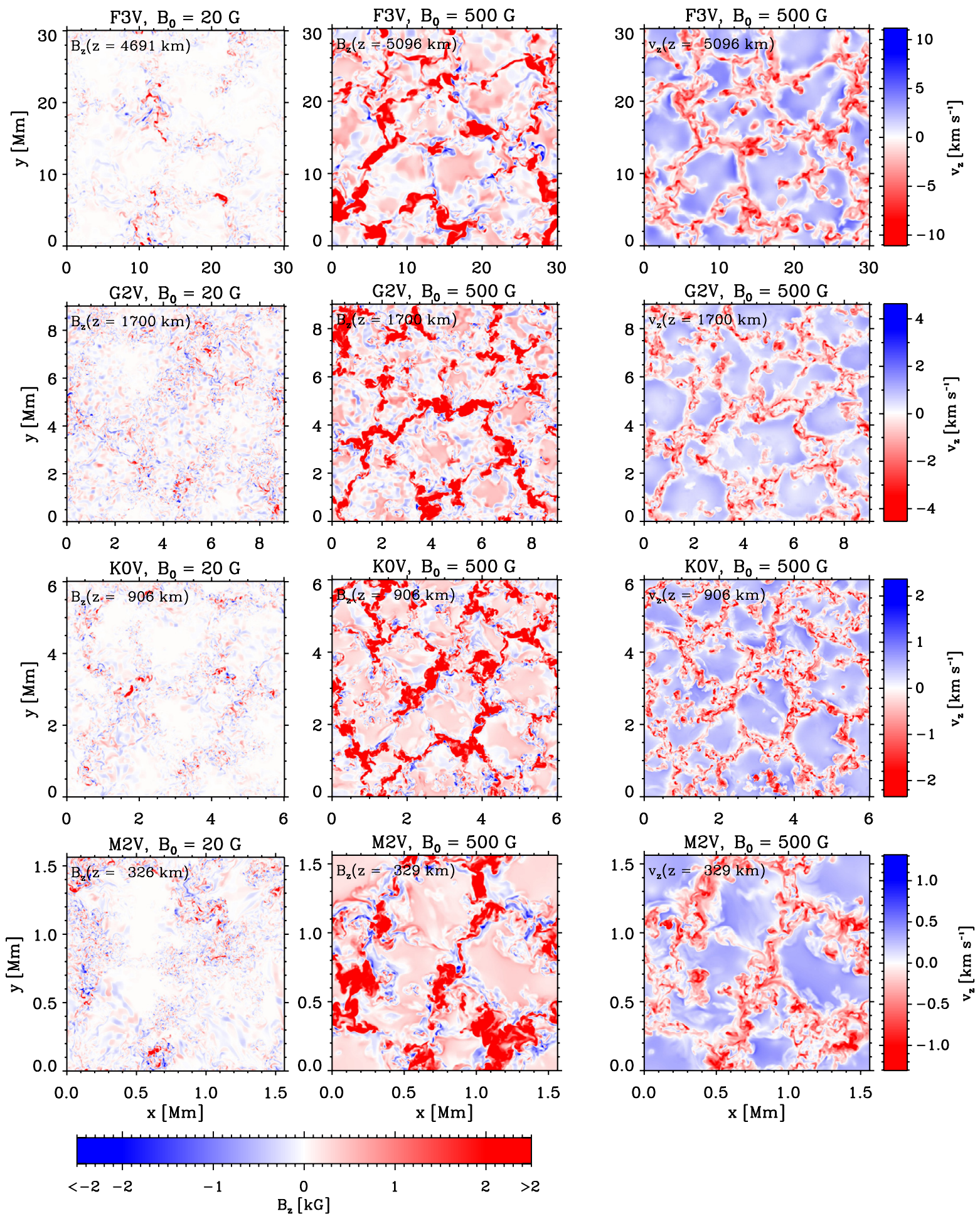

Fig. 10. Left two columns: maps of the vertical magnetic field component of the 20 and $500 \mathrm{G}$ runs of the F3V, G2V, K0V, and M2V simulations at a height corresponding to 4.6 pressure scale heights below the mean level of the optical surface. The geometrical depth $z$ of this level is specified in each panel. Right column: vertical component of the velocity of the $500 \mathrm{G}$ runs at the same depth.

place: horizontal outflows from the granules keep the magnetic flux in the downflow regions, while the upflows become nearly field-free. Owing to this process (in combination with the convective collapse), the field becomes locally very strong (several $\mathrm{kG}$ ) and, consequently, the magnetic pressure can locally surpass the turbulent and thermal pressures (see Sect. 5). As a consequence, the magnetic field reacts back on the velocity field.
Figure 10 displays maps of the magnetic field in a horizontal cut located 4.6 pressure scale heights below the optical surface for the $20 \mathrm{G}$ and $500 \mathrm{G}$ runs of the F3V, G2V, K0V and $\mathrm{M} 2 \mathrm{~V}$ stars. There are patches of both polarities. The dominating polarity (here shown in red) is concentrated in the downflows. Surrounding these are some regions of the opposite polarity, which are produced by field lines caught in the overturning 

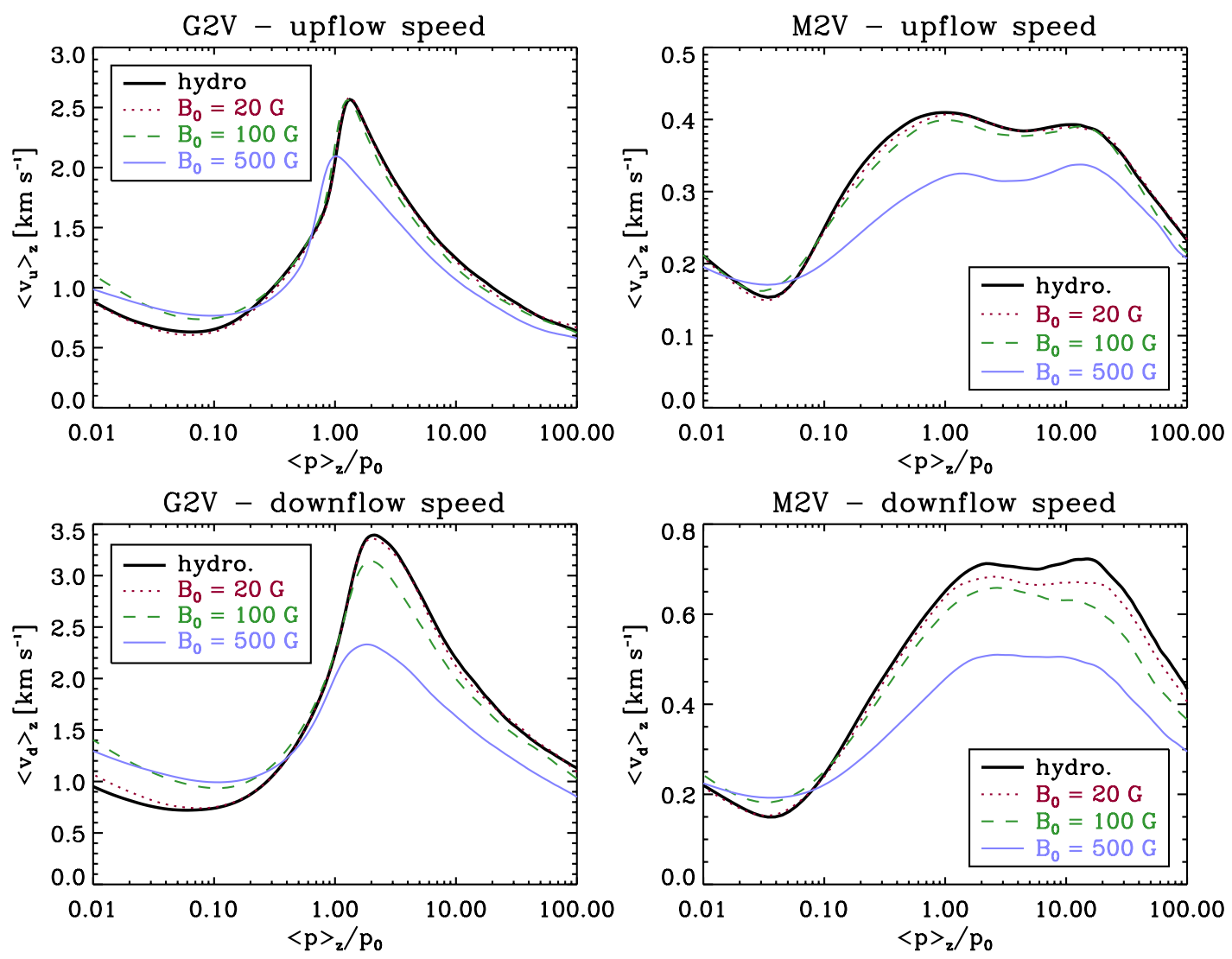

Fig. 11. Horizontal averages of the upflow (upper panels) and downflow (lower panels) speeds in the G2V (left panels) and M2V (right panels) simulations.

motion at the transition between up- and downflows. In the right column of the figure, the vertical velocity at the same depth is shown for the $500 \mathrm{G}$ run. As the mass supply in the magnetic flux concentrations from horizontal inflows is suppressed by the strong field, the flux concentrations prevent the formation of strong downdrafts below vertices of granules, which form in non-magnetic runs (see Paper I), and forces the downflows into a more network-like configuration around the magnetic flux concentrations and below unmagnetised intergranular lanes (cf. Nordlund \& Stein 1990).

Figure 11 illustrates the effect of the magnetic field on the up- and downflow speeds averaged over surfaces of constant geometrical depth $z$. As the magnetic field is concentrated in downflows, the effect of the magnetic field is stronger here. Below the surface, the downflows are slower in the magnetic runs than in the non-magnetic runs. This effect becomes stronger for increasing values of $B_{0}$. In the higher photosphere, the magnetic runs often show a higher average downflow speed. This can be interpreted as resulting from "convective collapse" events. The effect on the upflow speeds is qualitatively similar but smaller. It turns out that an average only over the weakly magnetised parts of the box (i.e. excluding the magnetic flux concentrations), shows no significant impact on the upflow speed near the optical surface whereas the downflow speed is still reduced by roughly $20 \%$ in the $500 \mathrm{G}$ runs. This shows that the effect on the upflows at the surface is only due to the reduction of the area which is available for normal convection, while the downflows are slower in the magnetic runs even in non-magnetic parts of the box. This is possibly a consequence of the more network-like structure of the downflows, which is more strongly affected by dissipation of kinetic energy than the efficient downdrafts in the non-magnetic runs.
We also find a change in the rms of the horizontal velocities, which are smaller below the optical surface by 35 to $40 \%$ in the $500 \mathrm{G}$ runs and up to twice as high in the upper photosphere in the $100 \mathrm{G}$ runs compared to the non-magnetic runs. The higher velocities in the upper photosphere can be explained by the magnetic coupling to lower layers (see Fig. 9).

Figure 12 shows 2D histograms of vertical velocity versus magnetic field strength in a layer of a width of half a pressure scale height both below and above $z=0$. The (kinematic) weakfield regime has a broad distribution of vertical velocities as the upflows and a part of the downflows are only weakly magnetised. At field strengths of up to a few $100 \mathrm{G}$, downflows are favoured. This is at the end of the kinematic regime, where the magnetic field is passively advected into downflow regions and is not yet strong enough to react back on the flows. These would include magnetic flux concentrations during their (early) collapse phase. At high field strengths of a few $\mathrm{kG}$, the distribution is rather narrow around zero velocity with a tendency towards downflows; however, the differences between spectral types are most obvious here: while the Sun and especially the F3V star still have a considerable spread in their velocities in the strong-field regime, magnetic flux concentrations are essentially at rest in the $\mathrm{K}$ and M stars. This probably is related to the different lifetimes of the magnetic flux concentrations: while in the hotter stars, the individual magnetic flux concentrations are short-lived and being constantly rearranged and fragmented by the surrounding flows, the magnetic structures in the $\mathrm{K}$ - and M-dwarf simulations are much longer-lived, so that most of them are in a stationary phase at any given time. This can be understood in terms of the balance between the turbulent pressure, the thermal gas pressure, and the magnetic pressure in the flux concentrations (see Table 3). Owing to the much larger ratio of (internal) magnetic pressure 

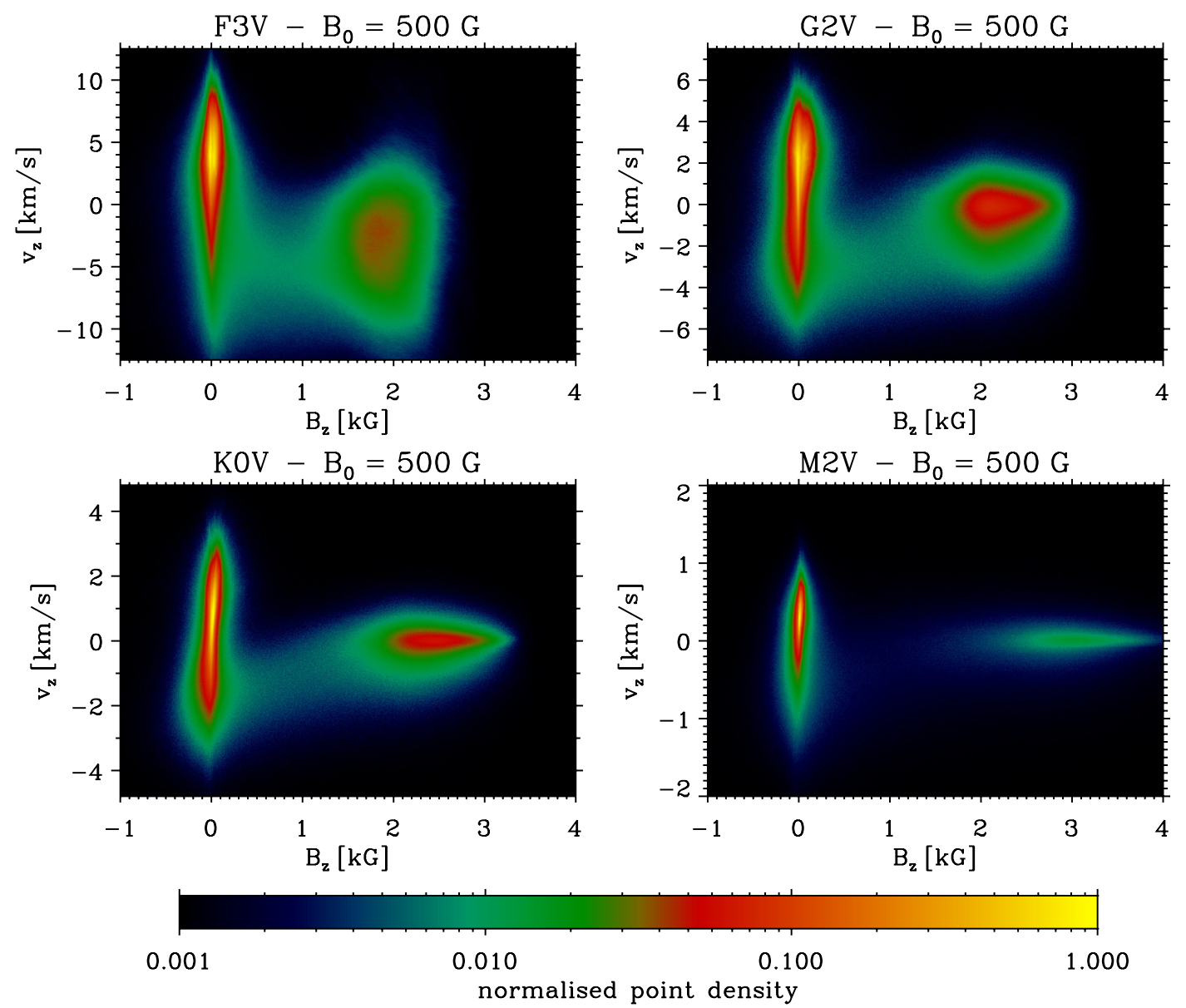

Fig. 12. Joint histograms of the vertical components of the magnetic field and the flow velocity in a layer between $\left\langle z\left(p=p_{0} \exp (-0.5)\right)\right\rangle$ and $\left\langle z\left(p=p_{0} \exp (+0.5)\right)\right\rangle$, where $p_{0}=\left\langle p\left(\tau_{\mathrm{R}}=1\right)\right\rangle$ is the mean pressure at the optical surface.

to (external) turbulent pressure in the magnetic flux concentrations of the cooler atmospheres of our sequence, the disruptive effects of the flows on the magnetic flux concentrations is much weaker.

As the only source of information on the magnetic field of stars are spectra (more precisely, profiles of spectral lines sensitive to the Zeeman effect), the relation between the magnetic and velocity fields becomes a problem: active regions on a star have an rms velocity differing from quiet regions resulting in a different velocity broadening of the spectral lines originating from differently magnetised surface components. Moreover, in our Fand G-star simulations, the magnetic field is mostly located in downflows, which adds a non-zero Doppler shift to the magnetically broadened line component. However, these effects are not taken into account at all when stellar magnetic fields are spectroscopically determined. A detailed analysis of the effect of the combined magnetic and velocity fields on a few spectral lines is given in Paper IV of this series.

\section{Conclusion}

Solar observations show that a substantial fraction of the observed magnetic flux is distributed in "plage" regions with a moderate average field strength of up to a few $100 \mathrm{G}$, which locally results in configurations very close to the one discussed in this paper. In these regions, magnetic bright points and faculae as well as (micro-)pores are observed, which are similar to the structures visible in our and other comprehensive 3D RMHD simulations of the Sun (see e.g. Carlsson et al. 2004; Keller et al. 2004; Cheung et al. 2008).
Here, we investigate how the appearance of moderately magnetised unipolar regions changes for a sequence of cool mainsequence stars (including the Sun). The major findings are:

- There is a lack of bright magnetic structures on M dwarfs (cf. Beeck et al. 2011). Owing to the more efficient convection (higher density near the optical surface, see Paper I) in these stars, the superadiabatic peak is less pronounced. This reduces the efficiency of field amplification by a convective collapse of thin flux tubes (cf. Rajaguru et al. 2002). The resulting Wilson depressions are shallow so that heating by radiation from the side walls becomes rather inefficient.

- The magnetic field strength in small-scale magnetic flux concentrations, $B_{\text {strong }}$, is almost independent of spectral type and of the total magnetic flux available. Along the mainsequence the thermal pressure at the optical surface increases with decreasing effective temperature. This compensates the lower efficiency of the convective collapse.

- For all spectral types considered, the upper photosphere is heated similarly by viscous and ohmic dissapation above small-scale magnetic field concentrations. The magnetic field dynamically couples the upper layers (where $p_{\text {mag }} \gg$ $\left.p_{\text {gas }}\right)$ to the deeper layers (where $p_{\text {mag }} \lesssim p_{\text {gas }}$ ).

- Convective flows are strongly modified within the magnetic flux concentrations. While the short-lived, smaller magnetic structures exhibit a strong downflow (convective collapse), the larger, long-lived structures are almost at rest as the inflows into the structures are blocked by the strong field.

We have shown that the spatial correlations between velocity field, thermodynamic variables, and the magnetic field 
B. Beeck et al.: 3D simulations of near-surface convection in main-sequence stars. III.

qualitatively and quantitatively differ between stellar types. The impact of this on the profiles of spectral lines in disc-integrated spectra, which are used for magnetic field measurements, is investigated in Paper IV of this series.

Acknowledgements. The authors acknowledge research funding by the Deutsche Forschungsgemeinschaft (DFG) under the grant SFB 963/1, project A16. A.R. has received research funding from the DFG under DFG $1664 / 9-2$.
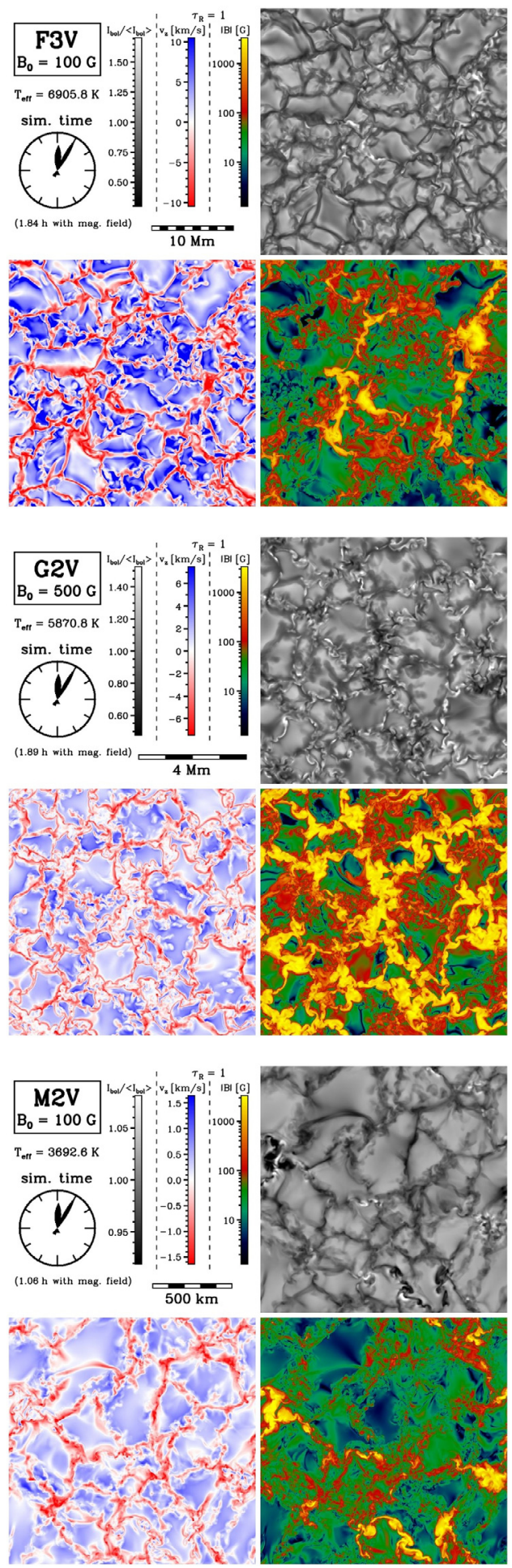

Fig. A.1. Snapshots of the six animations which are available as online material. Spectral type and $B_{0}$ are indicated in each panel.

The whole set of 18 movies can be downloaded from http://www2 . mps .mpg.de/projects/solar-mhd/movies2015/

\section{Appendix A: Animations of the magnetic simulation runs}

Movies illustrating the temporal evolution of six of the 18 magnetic simulation runs during the time span analysed in this paper and Paper IV are given as online material. They show the magnetic field strength and vertical velocity at the optical surface as well as the bolometric intensity. Figure A.1 provides snapshots of these six animations ${ }^{2}$.
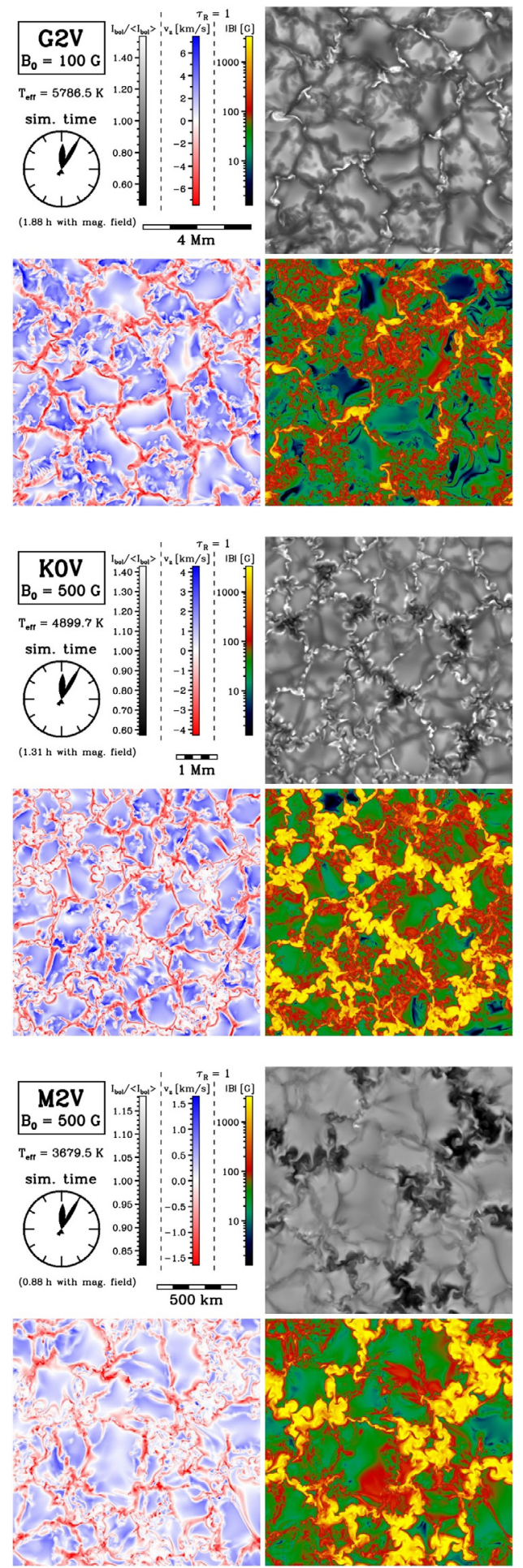


\section{References}

Beeck, B., Schüssler, M., \& Reiners, A. 2011, in 16th Cambridge Workshop on Cool Stars, Stellar Systems, and the Sun, eds. C. Johns-Krull, M. K. Browning, \& A. A. West, ASP Conf. Ser., 448, 1071

Beeck, B., Cameron, R. H., Reiners, A., \& Schüssler, M. 2013a, A\&A, 558, A48 Beeck, B., Cameron, R. H., Reiners, A., \& Schüssler, M. 2013b, A\&A, 558, A49 Beeck, B., Schüssler, M., Cameron, R. H., \& Reiners, A. 2015a, A\&A, 581, A43

Beeck, B., Schüssler, M., \& Reiners, A. 2015b, in Cambridge Workshop on Cool Stars, Stellar Systems, and the Sun 18, eds. G. T. van Belle, \& H. C. Harris, 467

Cameron, R., Schüssler, M., Vögler, A., \& Zakharov, V. 2007, A\&A, 474, 261

Carlsson, M., Stein, R. F., Nordlund, Å., \& Scharmer, G. B. 2004, ApJ, 610, L137

Charbonneau, P. 2010, Liv. Rev. Sol. Phys., 7

Cheung, M. C. M., Schüssler, M., Tarbell, T. D., \& Title, A. M. 2008, ApJ, 687, 1373

Cheung, M. C. M., Rempel, M., Title, A. M., \& Schüssler, M. 2010, ApJ, 720, 233

Fan, Y. 2009, Liv. Rev. Sol. Phys., 6, 4

Jafarzadeh, S., Solanki, S. K., Feller, A., et al. 2013, A\&A, 549, A116

Jeffers, S. V., Barnes, J. R., Jones, H., \& Pinfield, D. 2013, in Eur. Phys. J. Web Conf., 47, 9002

Keller, C. U., Schüssler, M., Vögler, A., \& Zakharov, V. 2004, ApJ, 607, L59

Kurucz, R. 1993, ATLAS9 Stellar Atmosphere Programs and $2 \mathrm{~km} \mathrm{~s}^{-1}$ grid, Kurucz CD-ROM (Cambridge, Mass.: Smithsonian Astrophysical Observatory), 13

Moll, R., Cameron, R. H., \& Schüssler, M. 2012, A\&A, 541, A68
Nordlund, Å., \& Stein, R. F. 1990, Comp. Phys. Comm., 59, 119

Petit, P., Dintrans, B., Solanki, S. K., et al. 2008, MNRAS, 388, 80

Pietarila Graham, J., Cameron, R., \& Schüssler, M. 2010, ApJ, 714, 1606

Rajaguru, S. P., Kurucz, R. L., \& Hasan, S. S. 2002, ApJ, 565, L101

Reiners, A. 2012, Liv. Rev. Sol. Phys., 9, 1

Reiners, A., \& Basri, G. 2007, ApJ, 656, 1121

Reiners, A., Schüssler, M., \& Passegger, V. M. 2014, ApJ, 794, 144

Reinhold, T., Reiners, A., \& Basri, G. 2013, A\&A, 560, A4

Rempel, M., Schüssler, M., \& Knölker, M. 2009a, ApJ, 691, 640

Schrijver, C. J., \& Zwaan, C. 2008, Solar and Stellar Magnetic Activity (Cambridge, UK: Cambridge University Press)

Schüssler, M. 2013, in IAU Symp. 294, eds. A. G. Kosovichev, E. de Gouveia Dal Pino, \& Y. Yan, 95

Shelyag, S., Cally, P. S., Reid, A., \& Mathioudakis, M. 2013, ApJ, 776, L4

Spruit, H. C. 1976, Sol. Phys., 50, 269

Stein, R. F. 2012, Liv. Rev. Sol. Phys., 9, 4

Stein, R. F., \& Nordlund, A. 2006, ApJ, 642, 1246

Stein, R. F., Lagerfjärd, A., Nordlund, A., \& Georgobiani, D. 2011, Sol. Phys., 268, 271

Steiner, O., Rajaguru, S. P., Vigeesh, G., et al. 2013, Mem. Soc. Astron. It. Suppl., 24, 100

Steiner, O., Salhab, R., Freytag, B., et al. 2014, PASJ, 66, 5

Stenflo, J. O. 2013, A\&ARv, 21, 66

Vidotto, A. A., Jardine, M., Morin, J., et al. 2013, A\&A, 557, A67

Vögler, A. 2003, Ph.D. Thesis, Georg-August-Universität Göttingen

Vögler, A., \& Schüssler, M. 2007, A\&A, 465, L43

Vögler, A., Shelyag, S., Schüssler, M., et al. 2005, A\&A, 429, 335

Wedemeyer, S., Ludwig, H.-G., \& Steiner, O. 2013, Astron. Nachr., 334, 137 